\title{
Evaluation of \\ capacitance-voltage characteristics for high voltage SiC-JFET
}

\author{
Tsuyoshi Funaki $^{1 a)}$, Tsunenobu Kimoto ${ }^{2}$, and Takashi Hikihara ${ }^{1}$ \\ ${ }^{1}$ Kyoto University, Dept. of Electrical Eng. \\ ${ }^{2}$ Kyoto University, Dept. of Electronic Science and Eng., \\ Graduate school of Engineering, Katsura, Kyoto, 615-8510, Japan
}

a)funaki@kuee.kyoto-u.ac.jp

\begin{abstract}
Capacitance between terminals of a power semiconductor device substantially affects on its switching operation. This paper presents a capacitance-voltage $(\mathrm{C}-\mathrm{V})$ characterization system for measuring high voltage $\mathrm{SiC}-\mathrm{JFET}$ and the results. The $\mathrm{C}-\mathrm{V}$ characterization system enables one to impose high drain-source voltage to the device and extracts the capacitance between two of three terminals in FET by eliminating its influence on the neighboring terminal. The capacitance between the gate and drain, and the drain and source represents the hybrid structure of the lateral channel and vertical drift layer of the SiC-JFET.
\end{abstract}

Keywords: $\mathrm{C}-\mathrm{V}$ characteristics, high voltage, $\mathrm{SiC}$, JFET, device structure

Classification: Electron devices

\section{References}

[1] J. L. Hudgins, G. S. Simin, E. Santi, and M. A. Khan, "An Assessment of Wide Bandgap Semiconductors for Power Devices," IEEE Trans. on PELS, vol. 18, no. 3, pp. 907-914, 2003.

[2] B. J. Baliga, "Silicon Carbide Power Devices," World Scientific Pub Co Inc, 2006.

[3] A. R. Hefner, Jr., R. Singh, J.-S. Lai, D. W. Berning, S. Bouche, and C. Chapuy, "SiC Power Diodes Provide Breakthrough Performance for a Wide Range of Applications," IEEE Trans. on PELS, vol. 16, no. 2, pp. 273-280, 2001.

[4] S. M. Sze, Physics of Semiconductor Devices (Second edition), John Wiley \& Sons, Inc., New York, 1981.

[5] T. Funaki, S. Matsuzaki, T. Kimoto, and T. Hikihara, "Characterization of punch-through phenomenon in SiC-SBD by capacitance-voltage measurement at high reverse bias voltage," IEICE Electron. Express, vol. 3, no. 16 , pp. 379-384, 2006.

[6] T. Funaki, J. C. Balda, J. Junghans, A. S. Kashyap, F. D. Barlow, H. A. Mantooth, T. Kimoto, and T. Hikihara, "SiC JFET de characteristics 
under extremely high ambient temperatures," IEICE Electron. Express, vol. 1, no. 17, pp. 523-527, 2004.

[7] T. Funaki, J. C. Balda, J. Junghans, A. Jangwanitlert, S. Mounce, F. D. Barlow, H. A. Mantooth, T. Kimoto, and T. Hikihara, "Switching characteristics of SiC JFET and Schottky diode in high-temperature dc-dc power converters," IEICE Electron. Express, vol. 2, no. 3, pp. 97-102, 2005.

[8] P. Friedrichs, H. Mitlehner, R. Kaltschmidt, U. Weinert, W. Bartsch, C. Hecht, K. O. Dohnke, B. Weis, and D. Stephani, "Static and Dynamic Characteristics of $4 \mathrm{H}-\mathrm{SiC}$ JFETs Designed for Different Blocking Categories," Materials Science Forum, vol. 338-342, pp. 1243-1246, 2000.

\section{Introduction}

Silicon carbide $(\mathrm{SiC})$ is a prospective semiconductor material for high voltage and high power switching devices for use in a switching power converter circuit $[1,2]$. A high-voltage SiC Schottky barrier diode (SBD) is commercially available, and various specifications of FET are under development [3]. The capacitance between terminals of a device affect the behavior of the device at switching operations. Therefore, it is necessary to simulate and analyze their behavior when designing switching power converter circuits. However, the capacitance in a semiconductor device changes nonlinearly with the applied voltage between terminals [4]; therefore, it must be characterized and modeled for analysis. The previous paper [5] presented the capacitance-voltage $(\mathrm{C}-\mathrm{V})$ characterization system for high voltage SiC SBD and clarified the occurrence of punch-through phenomena at the high voltage region. However, the characterization system cannot be applied to a FET, which has three terminals. Thus, this study develops capacitance measurement instrumentation to characterize high voltage $\mathrm{SiC}-$ JFET over a range of voltage ratings. Five circuit configurations are presented to measure the capacitance between the terminals of gate-source, gate-drain, drain-source, and input and output capacitance. The $\mathrm{C}-\mathrm{V}$ characteristics result from a hybrid structure of device are observed. This paper is one of the series of studies for characterization and modeling of $\mathrm{SiC}$ devices $[5,6,7]$.

\section{Characterization setup}

The cross-section of the high voltage SiC-JFET studied in this paper is given in Fig. 1 (a) [8]. It has hybrid structure of a lateral channel at the top of the device and a vertical drift layer for the body of the device. The depletion layer and the undepleted region of the semiconductor connected to electrodes constitute the capacitance of the device between terminals. There are three combinations of terminals that configure the capacitance of JFET. They are the gate-source $\left(C_{\mathrm{gs}}\right)$, gate-drain $\left(C_{\mathrm{gd}}\right)$, and drain-source $\left(C_{\mathrm{ds}}\right)$ capacitances, which are depicted in Fig. 1 (b). The thickness of the depletion layer changes with the applied voltage between terminals, and results in the change of 


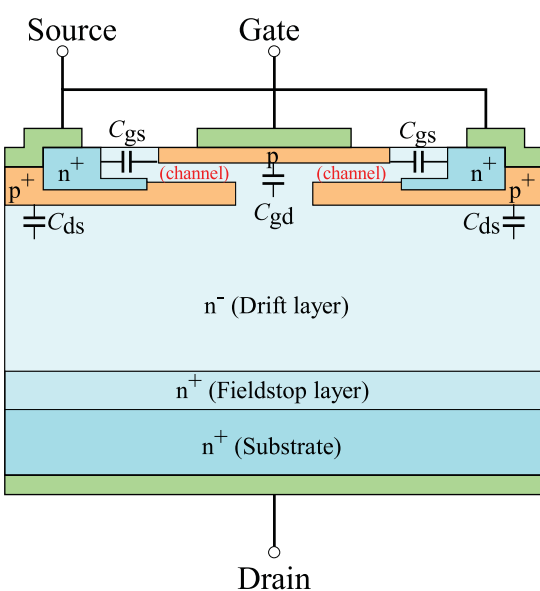

(a) Cross-section.

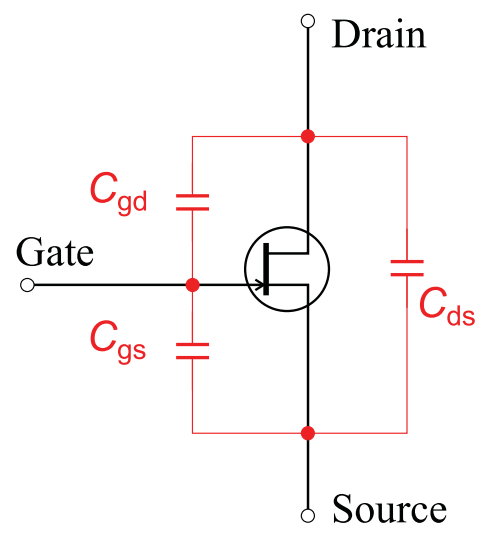

(b) Capacitance between terminals.

Fig. 1. High voltage SiC-JFET and capacitance between terminals.

capacitance. The JFET studied in this paper is normally on, and therefore, the measurement for capacitances requires blocking the channel of JFET by imposing a gate voltage. The $\mathrm{C}-\mathrm{V}$ characterization circuit given in Fig. 2 (a), which was presented in [5], can measure the capacitance by applying a bias voltage up to the device rated voltage. However, it does not have a circuit to supply gate voltage to the FET and cannot discriminate the capacitance between two terminals among three terminals. The additional circuits and fixtures are configured as follows. The following measurement procedures are based on a JEC-2405-2000 IGBT, but are refined to meet the high voltage ratings of the $\mathrm{SiC}$ device and extended to measure five capacitances.

Figure 2 (b) shows the circuit to measure $C_{\mathrm{ds}}$. The gate voltage $V_{\mathrm{gs}}$ to maintain the blocking condition of the device is supplied to the gate through a $100-\mathrm{k} \Omega$ resistance, which eliminates the influence of the $V_{\mathrm{gs}}$ source with a $1-\mu \mathrm{F}$ bypassing capacitor connected parallel to the voltage source. The gate terminal must be grounded in ac to exclude the influence of $C_{\mathrm{gs}}$ and $C_{\mathrm{gd}}$; therefore, a $1-\mu \mathrm{F}$ bypass capacitance is connected to the gate and grounded. The source terminal is isolated from the ground in ac, but grounded in dc through a $1-\mathrm{mH}$ blocking reactor. Then, $C_{\mathrm{ds}}$ is measured between the $\mathrm{H}^{\prime}$ and L' terminals by superimposing the drain-source bias voltage in the circuit shown in Fig. 2 (a).

Figure $2(\mathrm{c})$ shows the circuit to measure $C_{\mathrm{gd}}$. In this measurement, the gate terminal, which has a potential of $-V_{\mathrm{gs}}$ to the ground, must be connected to the $\mathrm{L}$ ' terminal for the measurement. Then, the $1-\mu \mathrm{F}$ decoupling capacitor is connected in series to the gate and the $\mathrm{L}$ ' terminal. The influence of $C_{\mathrm{gs}}$ and $C_{\mathrm{ds}}$ are excluded by connecting the source terminal to the ground. $C_{\mathrm{gd}}$ is measured between the H' and L' terminals by superimposing the drain-source voltage between the H' terminal and the ground.

Figure 2 (d) shows the circuit to measure $C_{\text {gs }}$. The drain-source bias voltage is imposed by the external voltage supply $V_{\mathrm{ds}}$, whose effect is eliminated by the high resistance connected in series and 1-nF bypass capacitor. This 


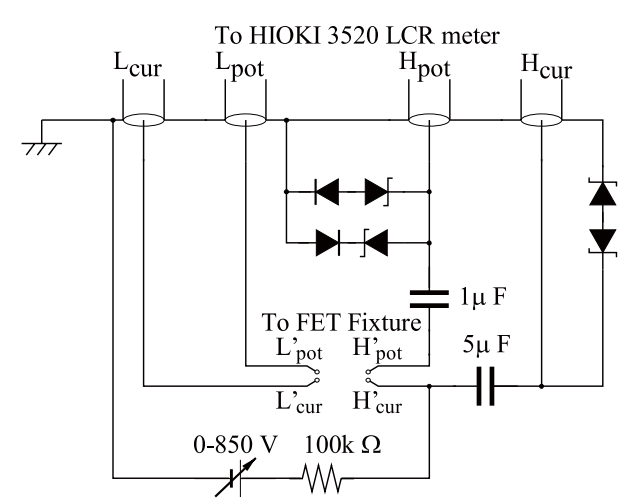

(a) Basic unit for high-voltage dc bias fixture.

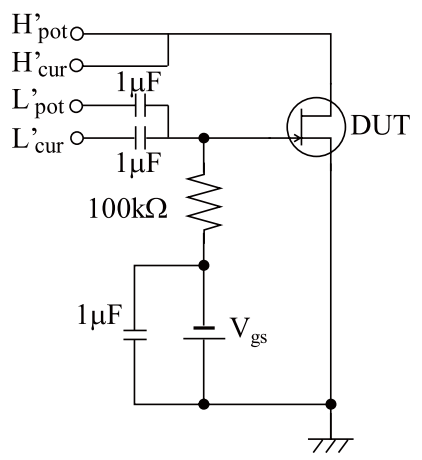

(c) $C_{\mathrm{gd}}\left(=C_{\mathrm{rss}}\right)$ measurement fixture.

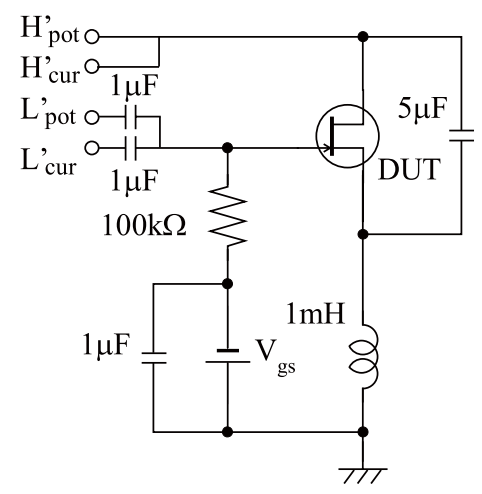

(e) $C_{\text {iss }}$ measurement fixture.

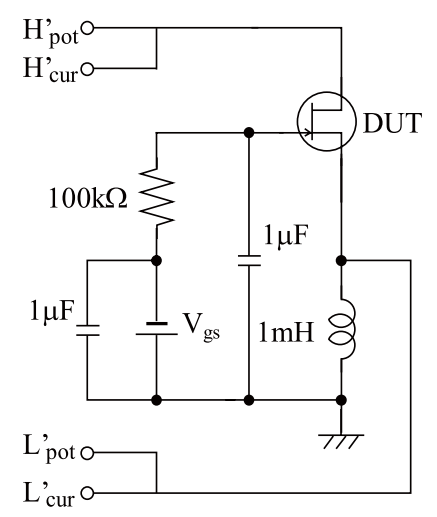

(b) $C_{\mathrm{ds}}$ measurement fixture.

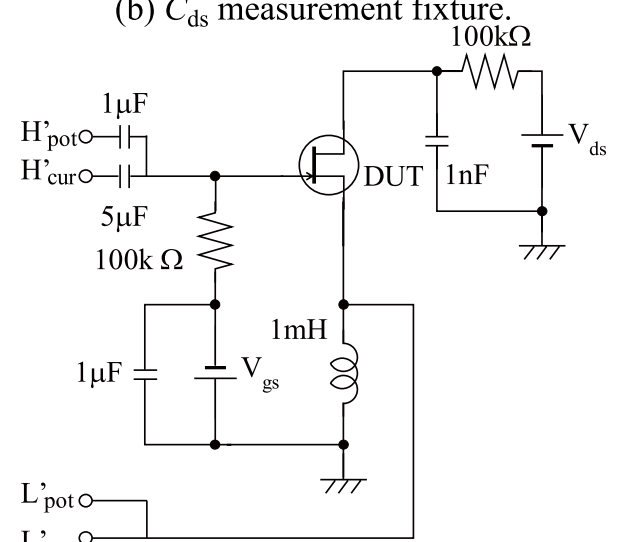

$\mathrm{L}_{\text {cur }}^{\prime}{ }^{\circ}$ (d) $C_{\mathrm{gs}}$ measurement fixture.

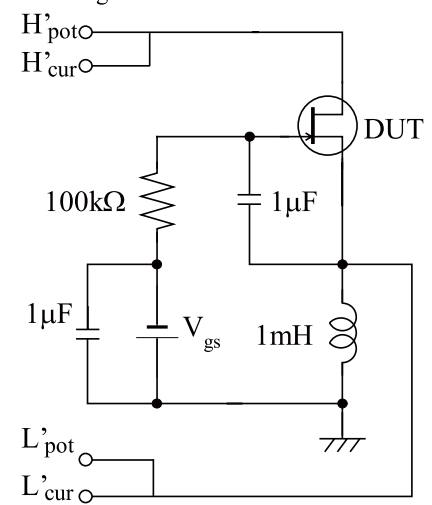

(f) $C_{\text {oss }}$ measurement fixture.

Fig. 2. C-V characterization setup for SiC-JFET.

capacitor also excludes the influence of $C_{\mathrm{gd}}$ and $C_{\mathrm{ds}}$.

The capacitance of a device can be sorted out into three specifications from the viewpoint of circuit behavior. They are the input capacitance $C_{\text {iss }}\left(=C_{\mathrm{gs}}+C_{\mathrm{gd}}\right)$, output capacitance $C_{\mathrm{oss}}\left(=C_{\mathrm{gd}}+C_{\mathrm{ds}}\right)$, and reverse transfer capacitance $C_{\mathrm{rss}}\left(=C_{\mathrm{gd}}\right)$. The measurement circuit for $C_{\mathrm{rss}}$ is same as $C_{\mathrm{gd}}$, which is shown in Fig. 2 (c). The circuit to measure $C_{\text {iss }}$ and $C_{\text {oss }}$ is as follows.

Figure $2(\mathrm{e})$ shows the circuit to measure $C_{\text {iss }}$. The drain and source are short-circuited in ac with a $5-\mu \mathrm{F}$ bypass capacitor and the influence of $C_{\mathrm{ds}}$ is eliminated.

Figure 2 (f) shows the circuit to measure $C_{\text {oss }}$. The gate and the source are short-circuited in ac with a $1-\mu \mathrm{F}$ bypass capacitor to eliminate the influence of $C_{\mathrm{gs}}$ by applying gate voltage $V_{\mathrm{gs}}$. 
The accuracy of the measured results are validated by pseudo FET capacitance consist of three capacitances with fixed value connected in delta, and confirmed that the errors arise from the fixtures are confined within $1 \%$.

\section{Results of measured C-V characteristics}

Figure 3 shows the measured $\mathrm{C}-\mathrm{V}$ characteristics of the studied $\mathrm{SiC}-\mathrm{JFET}$ with $2.4 \times 2.4-\mathrm{mm}$ in die size and $4-\mathrm{mm}^{2}$ active area, which is manufactured by SiCED GmbH. The measurements are performed with applying $V_{\mathrm{gs}}=-30 \mathrm{~V}$ to attain blocking condition of JFET. Fig. 3 (a) shows the linear plot of the measured $\mathrm{C}-\mathrm{V}$ result for the bias voltage range of $0<V_{\mathrm{ds}}<850 \mathrm{~V}$. It shows that $C_{\mathrm{gs}}$ has minute dependency on the drain-source voltage because it is the result of the depletion layer in the lateral channel region at the top of the device, as shown in Fig. 1(a). Therefore, the change in the thickness of the depletion layer at the drift layer to the variation of $V_{\mathrm{ds}}$ does not affect on $C_{\mathrm{gs}}$ for the pinched off condition of JFET. On the contrary, $C_{\mathrm{gd}}$ and $C_{\mathrm{ds}}$ change substantially with the variation of $V_{\mathrm{ds}}$ because of the change in the depletion layer thickness at the drift region. The measured $C_{\text {oss }}$ and $C_{\text {iss }}$ are approximately consistent with the sum of the respective constituent capacitances.

Figure 3 (b) shows the log-log plot of the measured $\mathrm{C}-\mathrm{V}$ characteristics. The non-smooth capacitance change in $C_{\mathrm{ds}}$ and $C_{\mathrm{oss}}$ around 50 to $70 \mathrm{pF}$ $\left(V_{\mathrm{ds}}=86 \mathrm{~V}\right)$ is due to the range switching in the LCR meter. The similar, but slight non-smooth change, is also found in $C_{\mathrm{gd}}$ for the same reason. $C_{\mathrm{gd}}$ and $C_{\mathrm{oss}}$ abruptly increase with the rise of $V_{\mathrm{ds}}$ to higher than $816 \mathrm{~V}$,

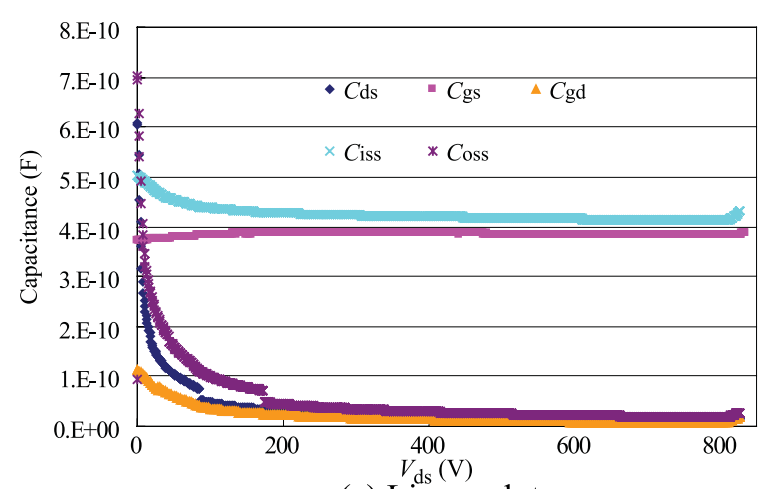

(a) Linear plot.

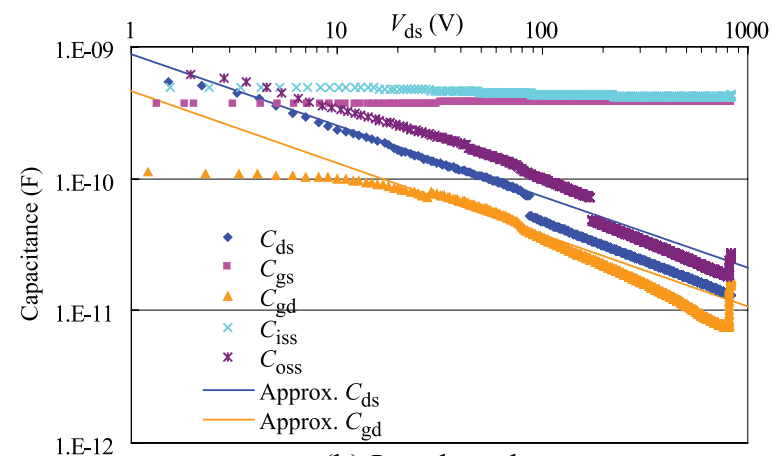

(b) Log-log plot.

Fig. 3. Measured C-V characteristics of SiC-JFET. 
which is caused by an avalanche breakdown of JFET for $V_{\mathrm{gs}}=-30 \mathrm{~V}$. The capacitance change does not saturate around this $V_{\mathrm{ds}}$, then the depletion layer does not reaches to the $\mathrm{n}^{+}$field stop layer. That is, reach through is not occurring. Then the breakdown is estimated to occur around $\mathrm{p}^{+}$source region, which is referred as punch through phenomenon in FET. The gradient of the $\mathrm{C}-\mathrm{V}$ curve in $C_{\mathrm{gd}}$ can be partitioned into two groups in the low and high voltage regions of $V_{\mathrm{ds}}$ around $V_{\mathrm{ds}} \cong 20 \mathrm{~V}$. The depletion region of $C_{\mathrm{ds}}$ expands from under the buried $\mathrm{p}^{+}$region to the drift layer in the low $V_{\mathrm{ds}}$ region. There is little room left in the expansion of the depletion region for $C_{\mathrm{gd}}$ at low $V_{\mathrm{ds}}$, because the depletion layer begins to expand from the channel opened between buried $\mathrm{p}^{+}$source region, as shown in Fig. 1 (a). Therefore, the variation of $C_{\mathrm{gd}}$ to $V_{\mathrm{ds}}$ change becomes less in the low $V_{\mathrm{ds}}$ region. The distribution of depletion layer is evened out by increasing the bias voltage. The $\mathrm{C}-\mathrm{V}$ relation can be approximated by Eq. (1), when the depletion layer is expanding to the whole drift area.

$$
C=C_{0}\left(V_{\mathrm{ds}}+V_{\mathrm{x}}\right)^{-m}
$$

Here, $C_{0}$ is the reference depletion capacitance, $V_{x}$ is the offset potential, and $m$ is the junction grading coefficient.

The coefficients are derived to be $m=0.54, C_{0}=470 \mathrm{pF}$ and $m=0.54$, $C_{0}=873 \mathrm{pF}$, respectively, for $C_{\mathrm{gd}}$ and $C_{\mathrm{ds}}$ in high $V_{\mathrm{ds}}$ region. The junction coefficients coincide for $C_{\mathrm{ds}}$ and $C_{\mathrm{gd}}$. Thus, they are clearly identified as drift layer characteristics.

\section{Conclusion}

This paper presents precise $\mathrm{C}-\mathrm{V}$ characteristics of high voltage $\mathrm{SiC}-\mathrm{JFET}$ to the respective combination of terminals for the rated range of drain-source voltage. To this end, the authors fabricated fixtures to measure the capacitance between terminals of JFET, which eliminates the influence of neighboring capacitances and can impose dc bias voltage up to the rated breakdown voltage of the device. The measured $\mathrm{C}-\mathrm{V}$ characteristics of JFET hold the hybrid structure of the studied SiC-JFET, which consists of a lateral channel and a vertical drift layer. The characteristics are as follows. $C_{\mathrm{gs}}$ is insensitive to the drain-source dc bias voltage and has a large constant value. The junction coefficients of $C_{\mathrm{gs}}$ and $C_{\mathrm{ds}}$ obtained from high dc bias voltage condition are identical due to depletion layer expansion to the uniformly doped drift layer. On the other hand, the expansion of depletion layer to the opened channel region at the top of the device is intricate and shows low capacitance variation at the low dc bias voltage region for $C_{\mathrm{gd}}$. The characterized $\mathrm{C}-\mathrm{V}$ properties of the device are crucial in modeling the SiC-JFET for circuit analysis.

\section{Acknowledgments}

This research was supported in part by the Ministry of Education, Culture, Sports, Sciences and Technology in Japan, the Grant-in-Aid for Sci- 
entific Research No. 17686024, 18360137, the 21st Century COE Program No. 14213201, and by Kansai Research Foundation for technology promotion (KRF). The authors would like to thank Mr. Matsuzaki of Kyoto University for assisting with the experiments. 